\title{
Variability in the size of the nucleus in spermatozoa from Houbara bustards, Chlamydotis undulata undulata
}

\author{
C. Lindsay ${ }^{1}$, H. J. Staines ${ }^{1}$, P. McCormick ${ }^{2}$, C. McCallum ${ }^{2}$, F. Choulani ${ }^{2}$ and G. J. Wishart ${ }^{\text {1* }}$ \\ ${ }^{1}$ Avian Reproduction Group, University of Abertay Dundee, Bell Street, Dundee DD1 1HG, UK; and ${ }^{2}$ International Foundation for \\ Conservation and Development of Wildlife, PO Box 116, Inezgane, Morocco
}

\begin{abstract}
Semen collected from 3-year-old male Houbara bustards contained large proportions $(6-40 \%)$ of spermatozoa with large nuclei. In these spermatozoa, the length of the nucleus was up to twice the mean length of the nucleus in normal spermatozoa. The lengths of the acrosome, midpiece and flagella were all normally distributed, but the length of the nucleus formed a bimodal distribution. The proportion of spermatozoa with large nuclei varied among males, but not among different semen samples collected from the same male throughout the breeding season. The proportion of motile spermatozoa with large nuclei was half that of normal spermatozoa, but their velocity was significantly greater. After insemination into females, spermatozoa with large nuclei were observed in the outer perivitelline layer of eggs laid, indicating that they were stored and transported within the oviduct and reached the egg at about the time of fertilization. Furthermore, there was no difference in the ability to produce viable progeny in females that were mated with males producing greater proportions of spermatozoa with large nuclei compared with those producing 'normal' spermatozoa. Thus, the abnormal spermatozoa did not appear to impede fertility. There were no signs of triploidy in the males that produced spermatozoa with large nuclei, or in their progeny, as demonstrated by the size of erythrocytes. Therefore, it appears that the spermatozoa with large nuclei were the result of aberrant spermatogenesis.
\end{abstract}

\section{Introduction}

Chlamydotis undulata undulata is one of three subspecies of the Houbara bustard, whose natural habitat lies from Mauritania to Egypt in North Africa. Habitat degradation, over-grazing by domestic animals and hunting have all played a significant role in the reduction of Houbara bustards (Collar, 1980). The species is listed under Appendix 1 of the Convention on International Trade in Endangered Species of Wild Fauna and Flora (CITES), which includes species threatened with extinction that are, or may be, affected by trade (CITES, 1997). It has also been classified as 'vulnerable' in the World Conservation Union's Red List of Threatened Animals (IUCN, 1994).

Therefore, attempts have been made to breed Houbara in captivity by natural mating (Mendlessohn $e t$ al., 1979) and, more successfully, by artificial insemination (Saint Jalme et al., 1994). The International Foundation for the Conservation and Development of Wildlife (IFCDW) in Morocco has developed a programme to study and carry out breeding of Houbara in captivity by artificial insemination and to reintroduce offspring to the wild. As part of this programme, semen was examined microscopically and was found to contain spermatozoa with nuclei of variable sizes. The aim of

${ }^{*}$ Correspondence.

Received 1 February 1999. this study was to describe the nature, statistical distribution and activity of these spermatozoa.

\section{Materials and Methods}

\section{Animals}

The founder breeding stock was obtained from egg harvests from local Houbara populations near Er Rachidia in Morocco during 1993 and 1994, under licence from La Direction des Fau et Forets et de la Conservation de Sols. During the non-breeding season, males and females were grouped in sex-specific groups. In January, the birds were moved to individual breeding pens $5 \mathrm{~m} \times 8 \mathrm{~m}$ and $2 \mathrm{~m} \times 16 \mathrm{~m}$ for males and females, respectively. The longitudinal configuration of pens allowed females to be in full visual contact with displaying males throughout the season. Normal watering and feeding arrangements continued throughout the breeding season and natural light was the only source of illumination for the birds. Most birds produced gametes by their second year and males that reached the documented breeding age of 3 years (Mendlessohn et al., 1979) in 1996 and 1997 were chosen for the present study. Female cages were checked for eggs and males were checked for display at least twice a day. 


\section{Semen collection}

Semen samples were collected once or twice a week throughout the breeding season between March and May. Nine 3-year-old males that provided semen regularly throughout the 1996 (birds A-E) and 1997 (birds F-I) breeding seasons were used for this study. These birds could be enticed to mount a dummy female bird, and were held by the operator while semen was collected onto a small Petri dish by intercepting cloacal contact with the dummy. The semen was transferred to a vial containing $200 \mu \mathrm{l}$ diluent 7.1, originally developed for turkey semen (constituents from Sigma-Aldrich and BDH, Poole; Lake and Ravie, 1982), at ambient temperature. The average semen volume and sperm concentration in all ejaculates from these birds was $77 \mu \mathrm{l}$ and $690 \times 10^{6}$, respectively.

\section{Semen analysis}

The morphology of spermatozoa was examined microscopically in smears stained with eosin and aniline blue (Haije, 1990) made up in diluent 7.1 (Lake and Ravie, 1982). Five microlitres of diluted semen was incubated with $40 \mu \mathrm{l}$ dye at $0^{\circ} \mathrm{C}$ for 2-3 $\mathrm{min}$ before the smears were prepared on standard microscope slides. At least 100 spermatozoa were examined under oil at $\times 1000$ magnification in duplicate smears from each sample. The length of individual sperm organelles was determined by automatic sizing after manual delineation of the length in digital images presented on a Quantimet-600 image analyser (Leica UK, Milton Keynes). Spermatozoa with 'large' nuclei were also identified by eye.

Sperm motility was also assessed semi-automatically with the Quantimet-600 image analyser. Five consecutive images, each $0.25 \mathrm{~s}$ apart, were captured and presented as a single image. The distances between each point were delineated with the cursor and the total distance travelled by the spermatozoa during $1 \mathrm{~s}$ (curvilinear velocity, VCL) was calculated automatically.

\section{Artificial insemination and fertility assessment}

Hens were inseminated with between 10 and $20 \times 10^{6}$ spermatozoa into the proximal vagina, which was revealed by manipulation of the cloaca with retractors. Only eggs laid by hens receiving a single insemination from one male were used for estimation of fertility. Egg fertility was assessed by illuminating the eggs ('candling') or breaking open eggs that did not hatch, to identify the presence of an embryo. Spermatozoa in the outer perivitelline layer were visualized by fluorescence microscopy after staining with diamidinophenylindole (DAPI) (Wishart, 1987).

\section{Erythrocyte analyses}

Blood was collected from the wing vein and smears were stained using Giemsa (BDH, Poole; Thorne et al., 1987). The length of the longest and shortest axes of the erythrocytes was measured as for the sperm organelles and the volume of the cells was calculated (Tiersch et al., 1991).

\section{Statistical analyses}

Simple linear regression techniques were used to test for time trends. Student's $t$ test or one-way ANOVA was used to test for differences in the mean values of subsets of the data and Tukey's pairwise comparison was used when such differences were present. The Ryan-Joiner test was used to determine whether the length of the sperm organelles was normally distributed. Unless otherwise stated, the 5\% significance level was used. All tests were performed using Minitab software (Microsoft, 1995).

\section{Results}

The morphology of three representative types of spermatozoon from a single ejaculate and the distribution of sizes of organelles in spermatozoa from the same ejaculate are shown (Figs 1 and 2, respectively). Normality plots derived from the Ryan-Joiner (RJ) test demonstrated that the lengths of the flagella $(R J=1.0 ; P>0.1)$, midpiece $(R J=1.0$; $P>0.1)$ and acrosome ( $R J=0.99 ; P=0.07)$ were all normally distributed, but that the length of the nucleus $(R J=0.80$; $P<0.01$ ) showed a longer tail at higher values than a normal distribution.

Measurements of the size of the nucleus in spermatozoa from each of three ejaculates from four males were also tested to determine whether they were normally distributed (Table 1). This was shown to be the case in all but two samples (bird B: 9/5, bird H: 10/5; Table 1) and enabled calculation of the proportion of spermatozoa outside of (greater than) this distribution. Since facilities for estimating the proportion of large spermatozoa by image analysis were not available for all samples, the effectiveness of subjectively identifying large sperm nuclei was tested in the same samples. The percentage of large nuclei estimated subjectively and those estimated objectively by image analysis in these samples is shown (Fig. 3). Although the objective estimations were generally higher than the subjective values, the results of the two methods showed a significant positive correlation (Fig. 3).

The proportion of spermatozoa with large nuclei in ejaculates from individual males did not vary significantly with time (each $P>0.05$ ) throughout the breeding season and the mean proportion per ejaculate is shown (Table 2). The mean proportions ranged between 5 and $40 \%$ and the differences among males were highly significant $(F(8,93)=$ $70.8 ; P<0.001$ ).

The mean percentage of motile spermatozoa from samples (from two different males) of diluted semen maintained at $40^{\circ} \mathrm{C}$ was 75.6 and $35.1 \%$ for normal spermatozoa and spermatozoa with large nuclei, respectively. The mean $( \pm \mathrm{SD})$ rates of motion for motile spermatozoa were $68.8 \pm 15.6$ $(n=14)$ and $90.6 \pm 14.9(n=42) \mu \mathrm{m} \mathrm{s}^{-1}$ for normal spermatozoa and spermatozoa with large nuclei, respectively, and were significantly different $(t(23)=4.70 ; P=0.0001$; Student's $t$ test). 

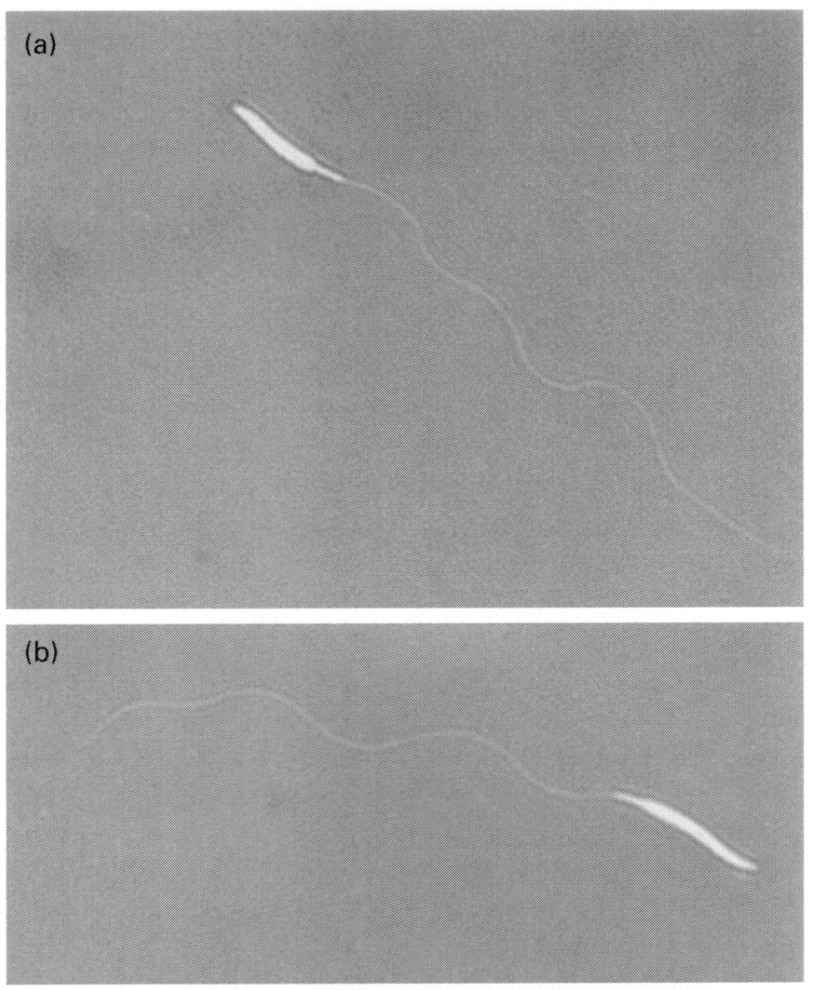

(c)

Fig. 1. Morphology of spermatozoa from Houbara bustard semen. (a) Normal spermatozoon; (b) spermatozoon with a large nucleus; (c) spermatozoon with a large nucleus and double flagellae. Scale bar represents $10 \mu \mathrm{m}$.

The ability of inseminated spermatozoa to be transported and stored within the oviduct was assessed by examining the size of the nucleus in spermatozoa that were trapped in the outer perivitelline layer of eggs laid (Fig. 4). The size distribution of nuclei in spermatozoa in dried smears of semen (from one male) before insemination and the size of DAPI-stained sperm nuclei trapped in the perivitelline layer of an egg laid by a hen inseminated with semen from the same male are shown (Fig. 5). Although there are some differences in size between the two samples (presumably due to the different procedures used for preparing the samples), there is a significant number of spermatozoa with large nuclei in the population in the perivitelline layer.

The proportion of fertile eggs laid by hens after a single insemination with samples of spermatozoa from eight of the nine birds is shown (Table 2). Large proportions of spermatozoa with large nuclei did not appear to compromise the fertilizing ability of these samples.

The volume $\left(\mu^{3}\right.$ mean $\left.\pm S D\right)$ of erythrocytes from birds $F$ and I, which produced the fewest and greatest number of spermatozoa with large nuclei during the 1997 season were $2284 \pm 298$ and $2170 \pm 264$, respectively, and were not significantly different (Student's $t$ test; $P>0.05$ ). The size of erythrocytes from three offspring of male I were $2419 \pm 353$, $2155 \pm 284$ and $2476 \pm 320$, respectively. Although these values are significantly different from those for bird $F$ (Student's $t$ test; $P<0.05$ ), the volume of erythrocytes in each of the five blood samples followed a normal distribution $(\mathrm{RJ}=1.00(P>0.1) ; 0.99(P>0.1) ; 0.98(P>0.07) ; 1.00(P>$ $0.1) ; 0.99(P>0.1)$ for birds $F, I$ and the three offspring, respectively).

\section{Discussion}

This study demonstrated that the semen of Houbara bustards (Chlamydotis undulata undulata) reared at IFCDW contains spermatozoa with nuclei that are greater in length than those in normal spermatozoa. The normal and large nuclei each formed a normal distribution; the large nuclei had a mean length twice that of the normal nuclei. The mean size of nuclei in these distributions also appeared to vary among individuals. The proportion of spermatozoa with large nuclei ranged from 5 to $40 \%$ among individuals, but did not vary among different ejaculates from the same individual within a breeding season. Although most of the routine estimations of the size of the sperm nuclei were performed subjectively, this system was validated by comparison with objective measurements by image analysis.

It is assumed that the greater size of the nuclei is indicative of a larger amount of DNA, as evidenced by the greater fluorescence of these spermatozoa after DAPI staining, as well as their appearance in smears stained with eosin and aniline. Although in mice, variation in the size of the sperm head is affected by different degrees of nuclear condensation (Pogany and Balhorn, 1992; Burruel et al., 1996), in rabbits, visualization of the size of the sperm head after staining with nigrosin and eosin is considered to be an adequate method of identifying diploid spermatozoa (Mortimer, 1977) and, in mice, variation in the nuclear morphology and DNA content of spermatozoa has been associated with irregular meiotic segregation (Stolla and Gropp, 1974). 'Normal' mammalian ejaculates contain different amounts of diploid or near diploid spermatozoa, for example, $1.97,0.10$ and $0.17 \%$ in semen from rabbits (Mortimer, 1977), bulls (Salisbury and Baker, 1966) and humans (Goldman et al., 1993), respectively. In the present study, spermatozoa with large nuclei were occasionally associated with other abnormalities, such as two flagellae, and showed intense DAPI staining over the entire length of the nucleus. Thus it is proposed that these spermatozoa contained more than the haploid content of genetic material and that some were actually diploid.

In normal semen of domesticated bird species (for example chickens, turkeys, duck, geese and quail), sperm nuclei are generally of a uniform length (Lake et al., 1968; see for example, Surai and Wishart, 1996), although turkey 
(a)

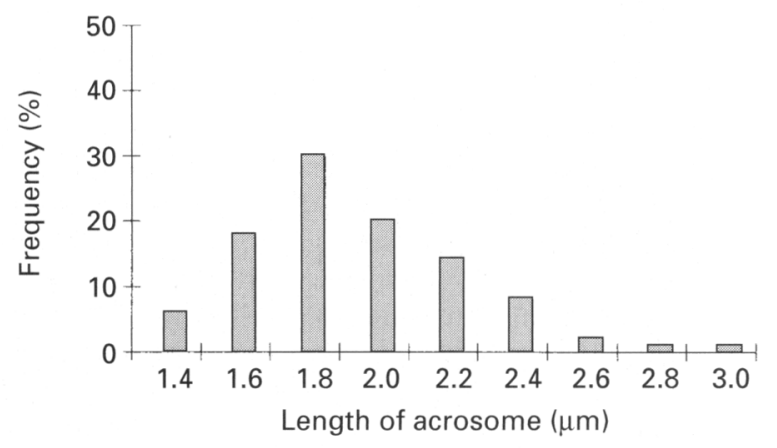

(c)

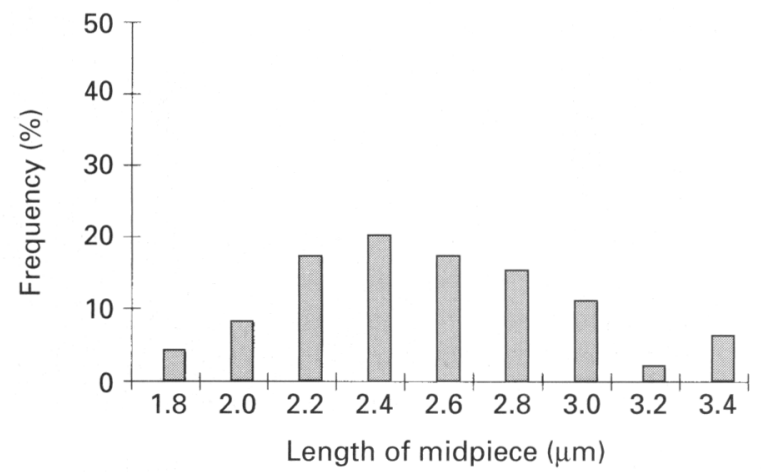

(b)

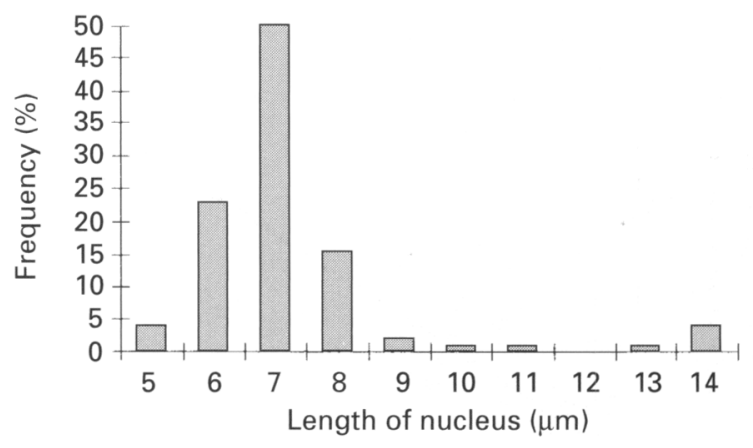

(d)

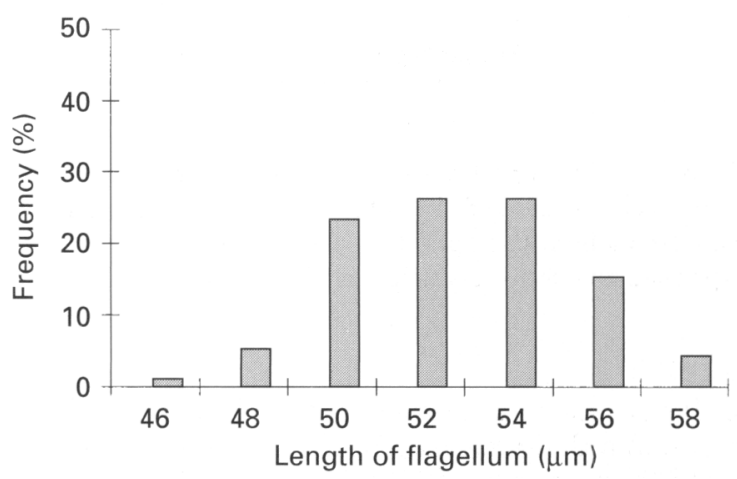

Fig. 2. Distribution of the length of (a) the acrosome, (b) the nucleus, (c) the midpiece and (d) the flagellum in spermatozoa from a single ejaculate from a 3-year-old male Houbara bustard. The mean lengths \pm SD were: $1.92 \pm 0.32 \mu \mathrm{m}$ for the acrosome; $2.54 \pm$ $0.38 \mu \mathrm{m}$ for the midpiece; $52.6 \pm 2.6 \mu \mathrm{m}$ for the flagellum; and $7.67 \pm 1.73 \mu \mathrm{m}$ for the nucleus; $n=100$ for all groups.

Table 1. Distribution parameters of three ejaculates from each of four male Houbara bustards

\begin{tabular}{|c|c|c|c|c|c|}
\hline \multirow{2}{*}{$\begin{array}{l}\text { Male } \\
\text { (year of birth) }\end{array}$} & \multirow{2}{*}{$\begin{array}{c}\text { Date of semen } \\
\text { collection } \\
\text { (day/month) }\end{array}$} & \multicolumn{3}{|c|}{$\begin{array}{l}\text { Mean and maximum values of normal } \\
\text { distribution of size of sperm nuclei }(\mu \mathrm{m})\end{array}$} & \multirow{2}{*}{$\begin{array}{r}\text { Percentage of } \\
\text { spermatozoa } \\
\text { with large nucle }\end{array}$} \\
\hline & & Mean & Maximum & $P$ value & \\
\hline \multirow[t]{3}{*}{ A (1993) } & $10 / 5$ & 5.70 & 7.54 & NS & 23 \\
\hline & $17 / 5$ & 5.92 & 8.09 & NS & 24 \\
\hline & $24 / 5$ & 5.61 & 7.03 & NS & 13 \\
\hline \multirow[t]{3}{*}{ B (1993) } & $23 / 4$ & 5.82 & 6.39 & NS & 9 \\
\hline & $9 / 5$ & 5.59 & 7.10 & 0.018 & 6 \\
\hline & $26 / 5$ & 5.16 & 7.27 & NS & 4 \\
\hline \multirow[t]{3}{*}{ C (1993) } & $2 / 5$ & 5.89 & 8.61 & NS & 3 \\
\hline & $9 / 5$ & 5.50 & 7.03 & NS & 4 \\
\hline & $1 / 6$ & 5.70 & 7.94 & NS & 8 \\
\hline \multirow[t]{3}{*}{ H (1994) } & $22 / 4$ & 7.27 & 10.01 & NS & 10 \\
\hline & $6 / 5$ & 7.44 & 11.06 & NS & 2 \\
\hline & $10 / 5$ & 7.64 & 10.16 & 0.025 & 6 \\
\hline
\end{tabular}

$P$ values are for the Ryan-Joiner test and the proportion of spermatozoa outside (greater than) this distribution is calculated as the percentage of spermatozoa with large nuclei.

NS, not significant.

spermatozoa with elongated nuclei (approximately 25\% greater in length) have been observed, albeit at an unknown frequency (Wakely and Kosin, 1951). Diploid spermatozoa from domestic fowl have large nuclei and can be induced at a frequency of $14-25 \%$ after treatment with colcemid to disrupt meiotic spindle formation during spermatogenesis (Wang et al., 1982). Furthermore, in certain strains of domestic fowl, triploidy occurs with a frequency of up to $20 \%$, apparently as a result of the production of diploid ova by non-disjunction at both meiosis I and meiosis II (Thorne and Sheldon, 1991), although fertilization by diploid spermatozoa has also been suggested as a cause (Zartman and Smith, 1975). The ZZW and ZZZ triploid males produced abnormal spermatids with one or two sets of 


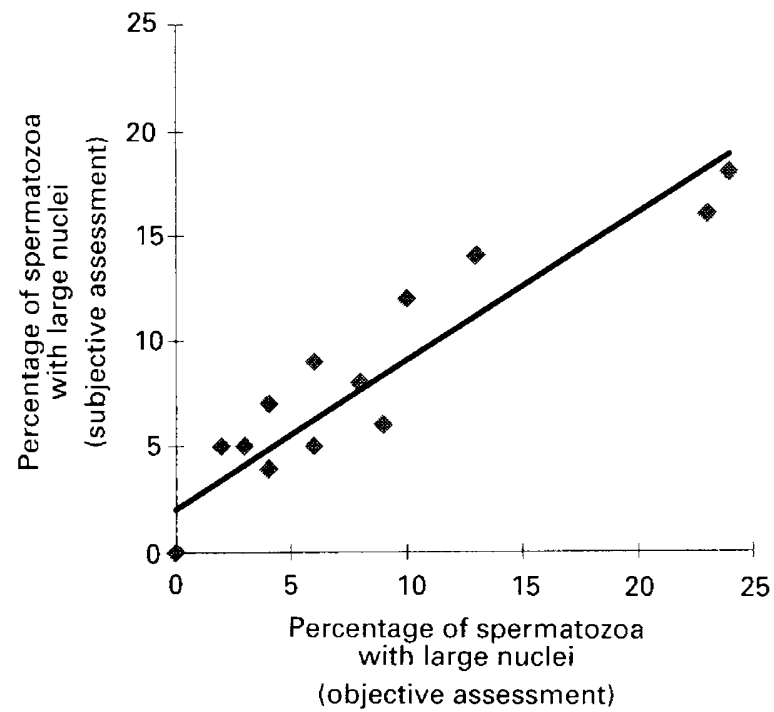

Fig. 3. Correlation of proportions of Houbara bustard spermatozoa with large nuclei estimated by objective image analysis and subjective examination. Each point represents an estimation made on 100 spermatozoa from a single ejaculate $(y=0.79 x+2.05 ; r=$ $0.93)$.

Table 2. Proportions of spermatozoa with large nuclei in ejaculates from individual male Houbara bustards over two seasons and the proportion of fertilized eggs laid by females inseminated by semen from these males

\begin{tabular}{lcc}
\hline Male & $\begin{array}{c}\text { Mean percentage of } \\
\text { spermatozoa with large } \\
\text { nuclei per ejaculate }(n)^{\mathrm{a}}\end{array}$ & $\begin{array}{c}\text { Proportion of } \\
\text { fertilized eggs }(n)^{\mathrm{b}}\end{array}$ \\
\hline A & $13 \pm 3(19)$ & $100(16)$ \\
B & $5 \pm 2(18)$ & $46(13)$ \\
C & $7 \pm 3(15)$ & $100(9)$ \\
D & $6 \pm 2(18)$ & $100(8)$ \\
E & $6 \pm 2(17)$ & $91(11)$ \\
F & $6 \pm 1(4)$ & $78(14)$ \\
G & $11 \pm 1(2)$ & - \\
H & $9 \pm 4(5)$ & $67(3)$ \\
I & $40 \pm 8(4)$ & $100(3)$ \\
\hline
\end{tabular}

Numbers in parentheses represent athe number of ejaculates and 'the number of eggs in which paternity is definitively assigned to each male.

organelles and large heads (Lin et al., 1995a) and the ZZZ males, in which spermatogenesis was less severely affected (Lin et al., 1995a), produced presumed diploid spermatozoa with heads that were 1.4-fold larger than haploid spermatozoa from normal $\mathrm{ZZ}$ males (Lin ct al., 1995b). Production of giant spermatozoa in dove-pigeon hybrids may also be the result of inappropriate chromosomal segregation (Smith, 1912). Triploid domestic fowl produce abnormally large erythrocytes that are approximately $25 \%$ greater in length than those from normal diploid birds (Abdel-Hameed et al., 1971; Thorne et al., 1987). Teirsch et al. (1991) reported the occurrence of triploidy, which is also associated with large erythrocytes, in parrots. This lead to the speculation that polyploidy is widespread in birds, since

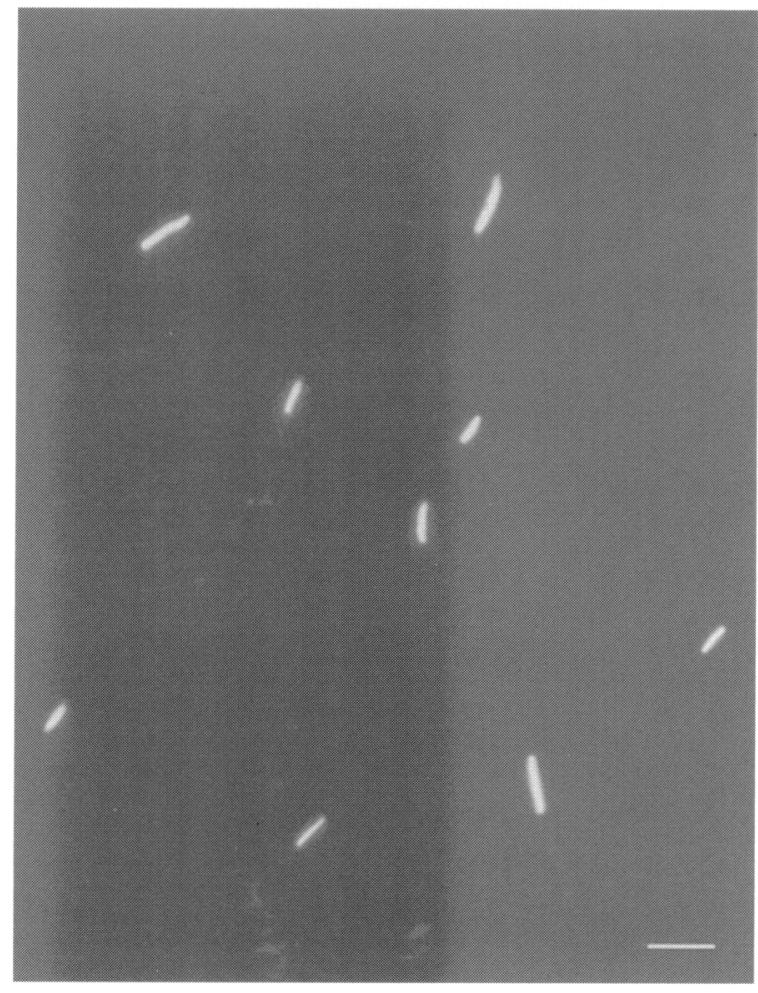

Fig. 4. Nuclei of Houbara bustard spermatozoa trapped in the outer perivitelline layer of an egg stained with diamidinophenylindole (DAPI). Scale bar represents $10 \mu \mathrm{m}$.

triploidy in domestic fowl is associated with intersexuality (Fechheimer, 1981) and intersexuality is widespread in sexually dimorphic species of bird (Taber, 1964). However, in the present study, there was no evidence of variation in the size of erythrocytes among males producing the greatest and fewest numbers of spermatozoa with large nuclei, or in the progeny of these males. Therefore, it appears that these individuals have normal ploidy and that the origin of the abnormal Houbara spermatozoa is meiotic disjunction.

Limited information is available on the incidence of sperm nuclei of different sizes in 'normal' wild species of birds. McFarlane (1971) studied 281 avian species, but did not note any intraspecific or individual differences in sperm morphology. Nwakolor et al. (1988) reported that approximately $10 \%$ of spermatozoa from exotic guinea fowl have primary abnormalities, which include large nuclei, but the actual proportions of these were not given. The spermatozoa with large nuclei found in Houbara semen appear to be similar to those present at an incidence of approximately $1 \%$ in semen from Sandhill cranes (Gee and Temple, 1978; Gee et al, 1985) and at 2.7\% in semen from Aleutian Canada geese (Gee and Sexton, 1990), although these studies did not report individual male differences or the functional characteristics of the spermatozoa. These proportions, and thus the presumed incidence of meiotic errors, are considerably smaller than those found in the present study in Houbara bustards.

In mammals, fertilization with an aneuploid spermatozoon would have serious implications for the subsequent 

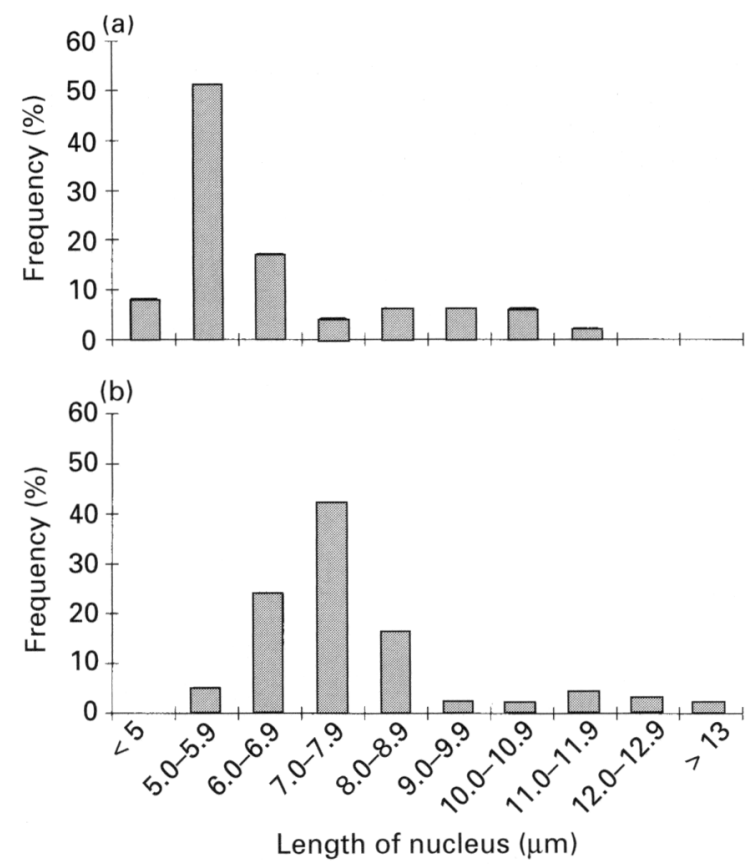

Fig. 5. Distribution of the length of the nucleus in Houbara bustard spermatozoa (a) in dried smears and (b) in the outer perivitelline layer of eggs laid by hens inseminated with semen from the same male.

development of the embryo, so it is fortunate that few diploid spermatozoa reach the site of fertilization (Mortimer, $1977,1979)$. In birds, polyspermy is normal and at least tens, and perhaps hundreds, of spermatozoa penetrate the germinal disc (Perry, 1987; Wishart, 1997). In the present study, significant numbers of spermatozoa with large nuclei were capable of reaching the site of fertilization, as indicated by the spermatozoa observed in the outer perivitelline layer of eggs laid. Since these spermatozoa are motile and have normal acrosomes, it is likely that they would be able to penetrate the inner perivitelline layer (Robertson et al., 1997). Fertility was not reduced in females inseminated with semen containing high proportions of spermatozoa with large nuclei. Furthermore, the resultant progeny, and all captive Houbara at IFCDW examined to date, appear to have normal ploidy. Therefore, it must be assumed that these abnormal spermatozoa are deselected at some stage of oocyte penetration or pronuclear formation and movement within the germinal disc, or that, during decondensation, a normal pronucleus is formed by discarding excess genetic material.

The authors thank HRH Prince Sultan bin Abdul Aziz Al Saud, Patron and Sponsor, for the facilities and funding to carry out the research, and Martin Maxwell for providing the blood smears.

\section{References}

Abdel-Hameed F, Neat HJ and Briles WE (1971) Differences in erythrocyte measurements of intersex and normal adult chickens Poultry Science $\mathbf{5 0}$ 1847-1854

Burruel VR, Yanagimachi R and Whitten WK (1996) Normal mice develop from oocytes injected with spermatozoa with grossly misshapen heads Biology of Reproduction 55 709-714
CITES (1997) Convention on International Trade in Endangered Species of Wild Flora and Fauna Appendices I and II http: / / www.wcmc.org.uk Collar NJ (1980) Bustards in decline British Birds 73 198-199

Fechheimer NS (1981) Origins of heteroploidy in chicken embryos Poultry Science $601363-1371$

Gee GF and Sexton TJ (1990) Cryogenic preservation of semen from the Aleutian Canada Goose (Branta canacensis leucopareia) Zoo Biology 9 361-371

Gee GF and Temple SA (1978) Artificial insemination for breeding nondomestic birds Symposia of the Zoological Society of London 43 51-72

Gee GF, Bakst MR and Sexton TJ (1985) Cryogenic preservation of semen from the Greater Sandhill Crane Joumai of Wildlife Management 49 480-484

Goldman AS, Fomina Z, Knights PA, Hill CJ, Walker AP and Hulten MA (1993) Analysis of the primary sex ratio, sex chromosome aneuploidy and diploidy in human sperm using dual-colour fluorescence in situ hybridisation European Journal of Human Genetics 1325-334

Haije U (1990) Evaluation and Cryopreservation of Fowl Semen (Gallus domesticus) Doctoral Thesis, University of Utrecht

IUCN (1994) The World Conservation Union, IUCN Red List of Threatened Animals. IUCN, Cambridge

Lake PE and Ravie O (1982) Effect on fertility of storing turkey semen for $24 \mathrm{hr}$ at $10^{\circ} \mathrm{C}$ in fluids of different $\mathrm{pH}$ British Poultry Science $2341-47$

Lake PE, Smith W and Young D (1968) The ultrastructure of the ejaculated fowl spermatozoon Quarteriy Journai of Experimental Physiology 53 356-366

Lin M, Thorne MH, Martin ICA, Sheldon BL and Jones RC (1995a) Electron microscopy of the seminiferous epithelium in the triploid ( $Z Z Z$ and $Z Z W$ ) fowl, Gallus domesticus. Journal of Anatomy 186563-576

Lin M, Thorne MH, Martin ICA, Sheldon BL and Jones RC (1995b) Development of the gonads in the triploid (ZZW and ZZZ) fowl, Gallus domesticus, and comparison with normal diploid males (ZZ) and females (ZW) Reproduction, Fertility and Development 7 1185-1197

McFarlane RW (1971) The Ultrastructure and Phylogenetic Significance of Avian Spermatozoa PhD Thesis, University of Florida

Mendelssohn H, Marder U and Stavy M (1979) Captive breeding of the Houbara bustard (Chlamydotis undulata macqueenii) and a description of its display International Council of Bird Preservation Bulletin XIII 134-149

Mortimer D (1977) The survival and transport to the site fertilization of diploid rabbit spermatozoa Journal of Reproduction and Fertility 51 99-104

Mortimer D (1979) Differential motility of diploid rabbit spermatozoa Archives of Andrology 2 41-47

Nwakolor LN, Okeke GC and Njoku DC (1988) Semen characteristics of the guinea fowl Numida numida meleagris. Theriogenology 29 545-554

Perry MM (1987) Nuclear events from fertilisation to the early cleavage stages in the domestic fowl (Gallus domesticus) Journal of Anatomy 150 99-109

Pogany GC and Balhorn R (1992) Quantitative fluorimetry of abnormal mouse sperm nuclei Journal of Reproduction and Fertility 96 25-34

Robertson L, Brown HL, Staines HJ and Wishart GJ (1997) Characterization and application of an avian spermatozoa:egg interaction assay using the inner perivitelline layer form laid chicken eggs Journal of Reproduction and Fertility 110 205-211

Saint Jalme M, Gaucher P and Paillat P (1994) Artificial insemination in Houbara bustard (Chlamydotis undulata): influence of the number of spermatozoa and insemination frequency on fertility and ability to hatch Journal of Reproduction and Fertility 100 93-103

Salisbury GW and Baker FN (1966) Frequency and occurrence of diploid bovine spermatozoa Journal of Reproduction and Fertility 11 477-480

Smith G (1912) Studies on the experimental analysis of sex Part 9. On spermatogenesis and the formation of giant spermatozoa in hybrid pigeons Quarterly Journal of Microscopical Science 58 159-170

Stolla R and Gropp A (1974) Variation of the DNA content of morphologically normal and abnormal spermatozoa in mice susceptible to irregular meiotic segregation journal of Reproduction and Fertility 38 335-346

Surai PF and Wishart GJ (1996) AI technology in the countries of the former USSR World's Poultry Science fournal $5227-43$

Taber E (1964) Intersexuality in birds. In Intersexuality in Vertebrates Including Man pp 285-310 Eds CN Armstrong and AJ Marshall. Academic Press, New York

Thorne MH and Sheldon BL (1991) Cytological evidence of maternal meiotic errors in a line of chickens with a high incidence of triploidy Cytogenetics and Cellular Genetics 57 206-210

Thorne MH, Collins RK and Sheldon BL (1987) Live haploid-diploid and other unusual mosaic chickens Cytogenetics and Cellular Genetics 45 21-25

Tiersch TR, Beck ML and Douglas M (1991) ZZW autotriploidy in a Blueand-Yellow Macaw Genetica 84 209-212

Wakely WJ and Kosin IL (1951) A study of the morphology of the turkey 
spermatozoa - with special reference to the seasonal prevalence of abnormal types Journal of the American Veterinary Association 44 240-245

Wang N, Sheppard JR, Wang T and Shoffner RN (1982) The induction and detection of diploid spermatozoa in Gallus domesticus. Mutation Research 73 279-290

Wishart GJ (1987) Regulation of the length of the fertile period in the domestic fowl by numbers of oviductal spermatozoa, as reflected by those trapped in laid eggs Journal of Reproduction and Fertility $80493-498$

Wishart GJ (1997) Quantitative aspects of sperm:egg interaction in chickens and turkeys Animal Reproduction Science 48 81-92

Zartman DL and Smith AL (1975) Triploidy and haploid-triploid mosaicism among chick embryos Cytogenetics and Cellular Genetics 15 138-145 